\title{
Simulation of Self-Neutralization Techniques for Charged Particle Thrusters
}

\author{
David C. Liaw*, Brian E. Gilchrist ${ }^{\dagger}$, and Thomas M. Liu \\ University of Michigan, Ann Arbor, MI, 48109, USA
}

\begin{abstract}
There is an emerging class of nanospacecraft thrusters that use colloids or nanoparticles that can be charged either positively or negatively to provide thrust. An issue to be examined is how the ability to charge particles to either polarity is beneficial to the system in terms of being able to provide self-neutralization of the thruster. Here, we focus on the capabilities of the nanoparticle field extraction thruster (NanoFET) to self-neutralize but our investigation applies to colloidal systems as well. We discuss two approaches for charged particle thruster neutralization using oppositely charged populations of nanoparticles - 1) spatially separated and 2) temporally separated - but focus mainly on the spatial approach. The majority of our exploration of these two neutralization options is done in XOOPIC ${ }^{T M}$, an object-oriented particle-in-cell simulation tool. From these simulations we see that self-neutralization using charged nanoparticles is not as trivial as it seems especially when compared to normal neutralization techniques using electrons as the neutralizing entity. This is because the equally weighted, massive nanoparticles are slow to move and neutralize the beam, resulting in image charge induced local electric field effects that negate portions of the spacecraft thrust. The significance of this degradation depends heavily on emitter characteristics such as emitter current and specific impulse.
\end{abstract}

\section{Nomenclature}

$\begin{array}{ll}\text { NanoFET } & \text { Nanoparticle Field Extraction Thruster } \\ q_{0} & \text { Charge Acquired by Metalized Nanoparticle, } \mathrm{C} \\ \varepsilon_{0} & \text { Electric Permittivity of Free Space: } 8.85^{*} 10^{-12} \mathrm{~F} / \mathrm{m} \\ E_{0} & \text { Charging Electric Field, } \mathrm{V} / \mathrm{m} \\ d & \text { Charging Gap, } \mathrm{m} \\ q & \text { Charge of Particle, } \mathrm{C} \\ E & \text { Background Electric Field, } \mathrm{V} / \mathrm{m} \\ F & \text { Force Exerted on Particle, } \mathrm{N} \\ m & \text { Mass of Particle, } \mathrm{kg} \\ a & \text { Acceleration of Particle, } \mathrm{m} / \mathrm{s}^{2} \\ d A & \text { Surface Area of Closed Surface, } \mathrm{m}^{2} \\ Q & \text { Enclosed Charge, C } \\ V & \text { Volume of Closed Surface, } \mathrm{m}^{3} \\ r & \text { Radius of Closed surface, } \mathrm{m} \\ t & \text { Time Taken for Spacecraft to Charge Up, } \mathrm{s} \\ I & \text { Current Emitted from the Closed Surface. A }\end{array}$

\footnotetext{
*Graduate Student Research Assistant, Electrical Engineering and Computer Science, 1301 Beal Ave, Ann Arbor, MI 48109, AIAA SS (student).

${ }^{\dagger}$ Professor, Atomspheric, Oceanic and Space Sciences and Electrical Engineering and Computer Science, 1301 Beal Ave, Ann Arbor, MI 48109, AIAA MC (associate fellow).

${ }^{\ddagger}$ Research Fellow, Aerospace Engineering, 1320 Beal Ave, Ann Arbor, MI 48109, AIAA MB (member).
} 


\section{Introduction}

An important consideration in the development of any electric propulsion system that emits charge is how the emitted beam as well as the spacecraft will be neutralized. Common electric propulsion systems such as ion thrusters and hall thrusters often use a hollow cathode neutralizer to neutralize the system. The hollow cathode neutralizer contains a hollow cylinder called an insert that is made of a material with a low work function, a heater wrapped around the insert that gets the insert up to the proper temperature, along with a cathode orifice plate, a reflective sheathing, and a keeper. The propellant, typically a gas, is flowed through the cathode, which causes electron-impaction ionization which creates a high-conductivity plasma cloud out in front of the emission area of the thruster. ${ }^{1}$ When positive charge is emitted from the thruster, the small, fast, mobile electrons from the plasma cloud are pulled along with the positive charge, neutralizing both the beam and the spacecraft. However, using a hollow cathode to neutralize adds a bit of complexity as it requires an additional component, but in this case, more importantly, it can decrease

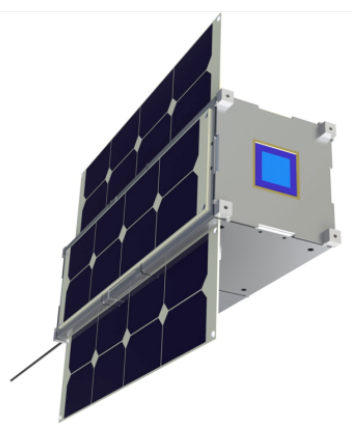

Figure 1. Visualization of the NanoFET system shown on a $3 U$ cubesat, with the blue square being the depiction of the NanoFET system. efficiency by over $20 \% .^{2}$

In addition to hollow cathode neutralizers, another potential neutralization device for microsatellites is a field emitter array cathode (FEAC). FEACs consist of an array of very sharp tips made of molybdenum or silicon, ${ }^{3}$ typically on the order of a few nanometers in curvature, and an extraction gate that is biased to a high positive potential creating an electric field on the order of $10^{9} \mathrm{~V} / \mathrm{m}$. This large electric field allows electrons to emit through electron tunneling from the conduction band through the solid-vacuum potential barrier. ${ }^{4}$ This electron emission can result in up to currents of up to $10 \mu \mathrm{A}$ per tip and the tips can have a density of $10^{6}$ tips $/ \mathrm{cm}^{2}{ }^{3}$ Although this would emit an acceptable number of electrons to neutralize, FEACs have not been shown to reliable in a harsh space environment. This is partially due to atomic oxygen being highly prevalent in lower earth orbit, which results in oxidation on the FEAC affecting its performance.

In the development of our NanoFET (Nanoparticle Field Extraction Thruster) system, we also need to consider the neutralization technique that we will be utilizing. Fig. 1 shows a visualization of what the NanoFET system would look like on a $3 \mathrm{U}$ cubesat, a $10 \mathrm{~cm}$ by $10 \mathrm{~cm}$ by $30 \mathrm{~cm}$ small spacecraft. Fig. 2 shows a visualization of the MEMS structure as well as the cutaway view of the NanoFET system. The NanoFET system uses a propellant of initially uncharged, metal-coated nanoparticles. These nanoparticles are stored in a particle reservoir, and when the system is to begin thrusting, there is a backpressure force applied to the particle reservoir pushing all the particles up to the charging sieve which is held at a high voltage.

Due to the construction of the charging sieve, only a single particle is allowed through each hole of the charging sieve at a time. Once a particle comes into contact with the charging sieve, it will undergo electrostatic charging. In the NanoFET system, a very large electric field is necessary to charge the particles up significantly as the charge acquired by the particle is directly proportional to the charging electric field as is shown in Eq. $1 .^{5}$

$$
q_{0}=\frac{\pi^{3}}{6} \varepsilon_{0} E_{0} d^{2}
$$

In our system, a possible charging electric field would have a $40 \mathrm{kV}$ voltage difference across a $100 \mathrm{um}$ gap, resulting in a $4 * 10^{8} \mathrm{~V} / \mathrm{m}$ electric field. At the moment that an impulse of thrust is desired, a piezoelectric is actuated, giving the additional axial force needed for the particles to lift off from the charging sieve, from which particles are attached due to the Van der Waals forces. ${ }^{6}$ These released, charged nanoparticles are then accelerated through the charging and the accelerating electric fields in order to continue to increase the particle's velocity. As the particles are emitted, the reactionary force on the spacecraft provides the spacecraft with its thrust. The NanoFET system envisions being able to emit particles with between 100 and $1000 \mathrm{~s}$ specific impulse depending on the charge to

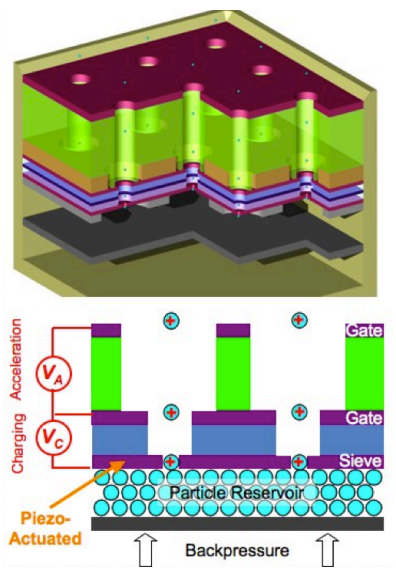

Figure 2. Visualization of the MEMS structure and cutaway view of the NanoFET system. Particles are stored in the particle reservoir, and with the pressure provided by the backpressure force are pushed to contact the charging sieve, which provides the nanoparticles with charge. Once the piezoelectric is actuated, particles lift off from the sieve and are accelerated across the charging and accelerating grid to provide thrust. 
mass ratio of the particles used as well as the charging and accelerating voltages.

For the NanoFET system, a neutralization technique still needs to be developed. In this case, a hollow cathode neutralizer such as the ones used by ion thrusters is even more complex because there would need to be an additional gas propellant to provide to the hollow cathode. Also, a FEAC would fit well with our micropropulsion system; however, it has not been shown to be capable of producing as expected in a harsh space environment as we envision NanoFET being suited for. However, one of the benefits of the NanoFET system is that it has the ability to charge particles both positively and negatively. This appears to provide us with a simple solution to this neutralization dilemma. The idea is that as long as we are able to emit equal amounts of positive and negative charge, the emitted beam will be net neutral and the spacecraft will remain net neutral as well.

\section{Neutralization Techniques}

Since we are attempting to emit equal amounts of positive and negative charge, there seems to be two logical neutralization techniques. The two are the spatial-varying, common time emission scheme as shown in Fig. 3 and the time-varying, common spatial emission scheme as shown in Fig. 4. In both schemes, the spacecraft wall is on the left, with particles being emitted out to the right.

In the spatial-varying, common time emission scheme, there are separate regions, each which only emits positive or negative charge. All regions will emit simultaneously, and as long as there are equal emission areas for positive and negative charge, and equal amounts of current coming out of the oppositely charged regions, there should be a net neutral beam and a net neutral spacecraft. In the timevarying, common spatial emission scheme, there is a single, large emission region, that emits only a single polarity of

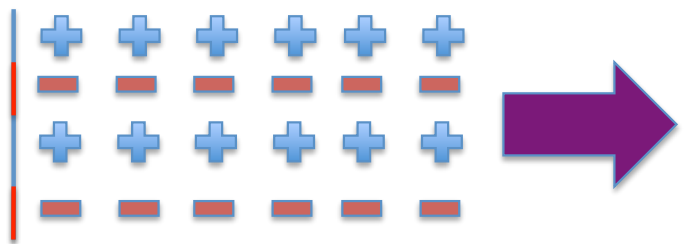
charged particle at any one time. However, this emission

Figure 3. Spatial-Varying, Common Time Emission Scheme region will vary between emitting positively and negatively charged particles. As long as the emission times and current are equal for the two polarities, the beam and spacecraft will be net neutral over time, with temporary charging of the beam and the spacecraft during polarity oscillations.

The main question that we would like to examine with these neutralization methods is whether NanoFET and other particle thrusters, such as colloidal thrusters, can utilize both positive and negatively charged particles to neutralize while maintaining performance.

\section{Methodology}

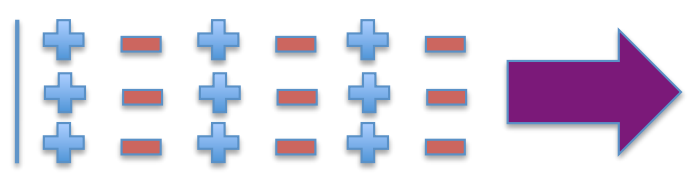

Figure 4. Time-Varying, Common Spatial Emission Scheme

The majority of our exploration of the two neutralization methods is done through simulation in $\mathrm{XOOPIC}^{T M}$. XOOPIC is a $2.5 \mathrm{D}$, object-oriented, particle-in-cell simulation tool. ${ }^{7,8}$ In XOOPIC, we are able to choose the simulation space, simulated particles, and simulated emitter. In the course of these simulations we use a Cartesian coordinate system, with particles emitted in the positive $\mathrm{x}$ direction, and the emitters moving in the $\mathrm{y}$ direction. In this case we have an $\mathrm{x}$-distance of $0.2 \mathrm{~m}$ and a y-distance of $0.15 \mathrm{~m}$. In the past we have also conducted simulations using a cylindrical coordinate system; however, in such cases it is much more difficult to maintain symmetry between the positively and negatively charged beams while keeping equal emitter areas.

We simulate two different types of particles. The more common nanoparticle that we simulate is a 200 $\mathrm{nm}$ solid polystyrene particle (assume metalized) that we intend to use in the NanoFET system. These particles have a mass of $4.4^{*} 10^{-18} \mathrm{~kg}$ and obtain a charge of $7.32^{*} 10^{-16} \mathrm{C}$ based on a charging electric field of $4^{*} 10^{8} \mathrm{~V} / \mathrm{m} .{ }^{6}$ These particles are approximately seven orders of magnitude heavier than a normal Xe+ ion and contains about 4500 elementary charges. This mass and charge translates to approximately a $100 \mathrm{C} / \mathrm{kg}$ charge to mass ratio. This is significantly lower than the specific charge of both electrons and Xe+ ions, which means that these particles that we are simulating are much heavier compared to the charge that they 
contain compared to normal neutralizing electrons. The other particle that we simulate is a $50 \mathrm{~nm}$ hollow polystyrene particle (assume metalized). These are particles that we are attempting to develop for use in the NanoFET system. These particles have a mass of $4.45^{*} 10^{-20} \mathrm{~kg}$ and a charge of $4.46^{*} 10^{-17} \mathrm{C}$ based on a charging electric field of $4^{*} 10^{8} \mathrm{~V} / \mathrm{m}$ translating to a charge to mass ratio of approximately $1000 \mathrm{C} / \mathrm{kg}$. Thus, these particles act slightly more like the common neutralizing electrons.

The emitter that we simulate for the most part emits particles at a current density of $0.967 \mathrm{~A} / \mathrm{m}^{2}$ for each emitter beam. This is compared to approximately $680 \mathrm{~A} / \mathrm{m}^{2}$ current density for our emitter as determined by the classical space charge limit calculated using the Child-Langmuir Law $^{9}$ and the three-dimensional ratio. ${ }^{10}$ Although NanoFET is envisioned to have the capability to emit particles at between 100 and $1000 \mathrm{~s}$ specific impulse, our simulations will concentrate on the upper limit as an initial condition. As we drop specific impulse from $1000 \mathrm{~s}$ to $100 \mathrm{~s}$ specific impulse, the space charge limit will also decrease and we anticipate seeing more detrimental effects to our spacecraft, but this is something that needs continued exploration. With a $1000 \mathrm{~s}$ specific impulse, we will be emitting particles at approximately $10^{4} \mathrm{~m} / \mathrm{s}$. In addition, particles are coming out with a temperature of approximately $0.03 \mathrm{eV}$, which is extremely cold.

The issues that we will examine in XOOPIC are whether there are local effects near the emitter that we should be concerned about and whether non-neutral beams are significantly disrupted and when it is of concern. In both schemes there will be regions of localized charge, and because of that there will be an image charge induced on the spacecraft wall of the opposite polarity. This causes an image charge induced electric field that is either pointing towards or away from the spacecraft depending on the polarity of the emitted particles. Either way, this image charge induced electric field will slow down the particles velocity as well as negate some of the thrust of the spacecraft due to the emission of these particles. We need to explore what kind of effect this image charge induced electric field has, and how we can mitigate such an effect. In addition, both schemes will emit oppositely charged particles, resulting in convergence of the oppositely charged particles towards one another. We also need to explore what will happen when these oppositely charged particles collide.

\section{Spatial-Varying, Common Time Emission Scheme}

In the following simulations, the four diagnostics being shown are X vs Y Particle Propagation, Electric Field, Velocity in the Y-direction with respect to X-position, and Velocity in the X-direction with respect to X-position. The X vs Y particle propagation diagnostic shows the simulation space with the spacecraft wall being simulated on the left (y-axis) and particles being emitted out to the right. In all the simulations shown, particles have not quite reached the right edge of the simulation space and thus are not subjected to any edge effects that may occur. However, we have conducted a set of simulations that are not shown that prove that the major effects that are observed in the shown simulations are not transient effects, and exist in steady state as well and are continuing to explore what differences do exist in the transient states, if any. In addition, as will be obvious in the particle propagation diagnostics, there are no collisions being modeled in this XOOPIC code that we are using. This is something that needs to be simulated as well; however, we do not anticipate any significant issues due to collisions, as they occur far enough away from the emitter that we do not anticipate scattering to come back to the spacecraft. The velocity in the y-direction plots show velocity in the vertical direction primarily between the two beams, and the velocity in the $\mathrm{x}$-direction plots show velocity in the horizontal direction away from the emitter.

\section{IV.A. Time Progression of 200 nm Solid Polystyrene Particles}

First we examine the spatial-varying, common time emission scheme that we introduced earlier. Again, this is the scheme that has separate regions, each of which emit only positive or negative charge simultaneously. Our first simulation looks at how the image charge induced electric field as well as the velocities of the particles change as nanoparticle beams are emitted. In this simulation we will be emitting $200 \mathrm{~nm}$ solid polystyrene particles that have a mass of $4.4^{*} 10^{-18} \mathrm{~kg}$ and contain $7.32^{*} 10^{-16} \mathrm{C}$ of charge. These particles are emitted at $1000 \mathrm{~s}$ specific impulse and $0.03 \mathrm{eV}$. We are emitting two beams of oppositely charged particles positioned next to one another. Each of these emitters is $1.5 \mathrm{~cm}$ in width and emitting $14.5 \mathrm{~mA}$ of current resulting in a current density of $0.967 \mathrm{~A} / \mathrm{m}^{2}$, which means that there will be equal amounts of positive and negative charge emitted, resulting in a beam that has no overall charge. This current density is well below the space charge limit of $680 \mathrm{~A} / \mathrm{m}^{2}$ in this case. Fig. 5 and Fig. 6 show the propagation of this beam when 
the particles are $25 \%$ of the way across the simulation space, and right before the particles get to the end of the simulation space.

$X$ vs Y Particle Propagation Resulting Velocity $\left(V_{Y}\right.$ vs $\left.X\right)$ in $\mathrm{m} / \mathrm{s}$
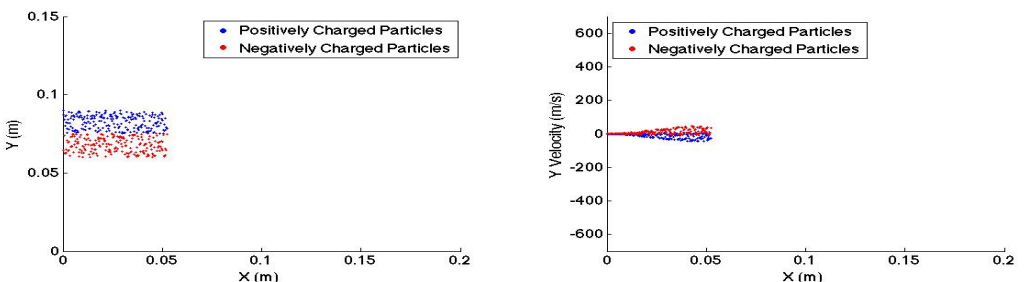

Electric Field

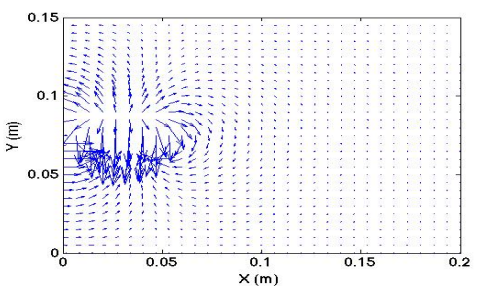

Resulting Velocity $\left(V_{x}\right.$ vs $\left.X\right)$ in $\mathrm{m} / \mathrm{s}$

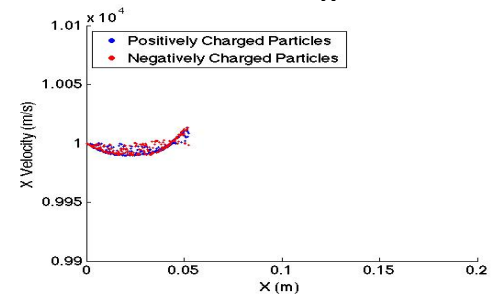

Figure 5. Beam Propagation of $200 \mathrm{~nm}$ Solid Polystyrene Particles at Early Time Stage

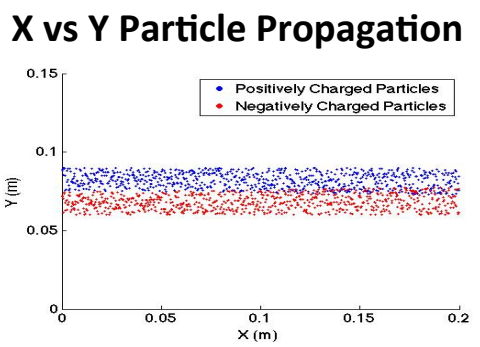

Resulting Velocity $\left(V_{Y}\right.$ vs $\left.X\right)$ in $\mathrm{m} / \mathrm{s}$

Electric Field

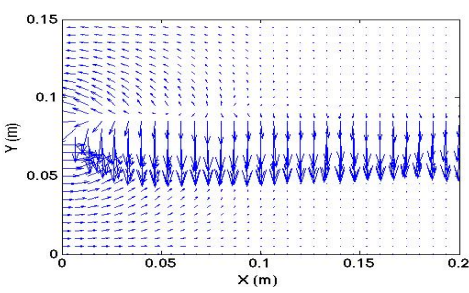

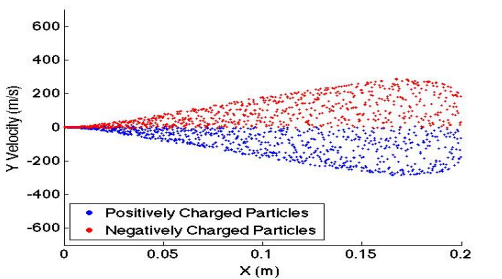

Resulting Velocity $\left(V_{x}\right.$ vs $\left.X\right)$ in $\mathrm{m} / \mathrm{s}$

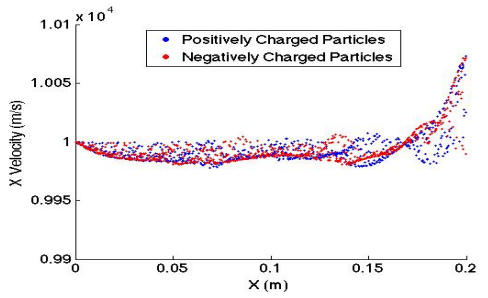

Figure 6. Beam Propagation of 200 nm Solid Polystyrene Particles at Final Time Stage

From these images we can see that as the beams continue to propagate across the screen, they tend to slowly converge towards one another and overlap because they are oppositely charged. We can see that the radial electric field draws the beams slowly on top of one another over the $0.2 \mathrm{~m}$ of the simulation space. This slow convergence is not a concern for performance; however, this convergence illustrates that the massive, equally massed particles do not neutralize as rapidly as we had envisioned unlike neutralizing electrons. Also we can see that the image charge induced axial electric field local to the emitter causes a decrease in velocity resulting in a maximum velocity drop of $0.22 \%$ relative to the initial emission velocity. This maximum decrease in velocity will continue to increase as the current density continues to increase towards the space charge limit. We can also see that as time progresses the electric field gets stronger and stronger, resulting in the stronger convergence between the two beams at the final time stage, along with a larger drop in velocity. We also notice that there is an increase in velocity, even above the original velocity, on the leading edge of the beam. This is due to all the like charged particles behind the front edge accelerating it forward. 


\section{IV.B. Time Progression of 50 nm Hollow Polystyrene Particles}

The next set of simulations we ran shows the time progression of a net neutral emitted beam with $50 \mathrm{~nm}$ hollow polystyrene particles rather than $200 \mathrm{~nm}$ solid polystyrene particles. We are currently looking to develop these particles for use in the NanoFET system. The particles have a mass of $4.45^{*} 10^{-20} \mathrm{~kg}$ and contain a charge of $4.46^{*} 10^{-17} \mathrm{C}$. This results in approximately a $1000 \mathrm{C} / \mathrm{kg}$ charge to mass ratio, which is ten times higher than the specific charge in the previous simulation. All other emitter and simulation space parameters are the same as the previous simulation. Since the charge on each particle is less than the previous simulation, and the current density is kept the same, there will be more particles emitted; however, in our simulation, it will appear as if there are similar numbers of particles emitted between this and the previous simulation. This is because in particle in cell simulations, oftentimes numerous particles will be represented by a single superparticle, and in this case we keep the number of simulated superparticles the same and this will not change any of the physics. Fig. 7 and Fig. 8 show the progression of this beam at two time steps.

\section{$X$ vs Y Particle Propagation Resulting Velocity $\left(V_{Y} v s X\right)$ in $\mathrm{m} / \mathrm{s}$}
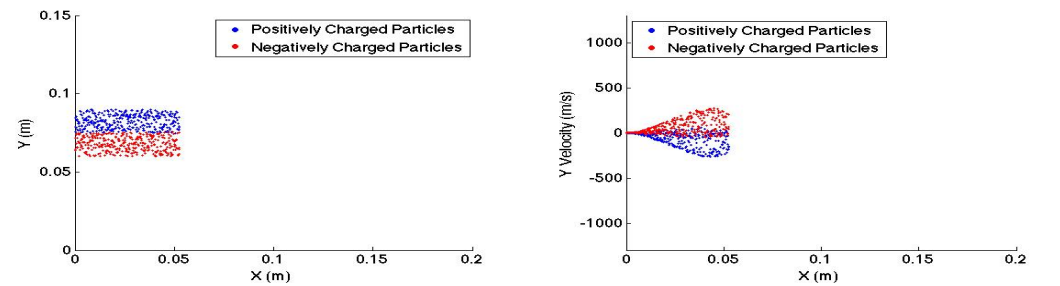

Electric Field
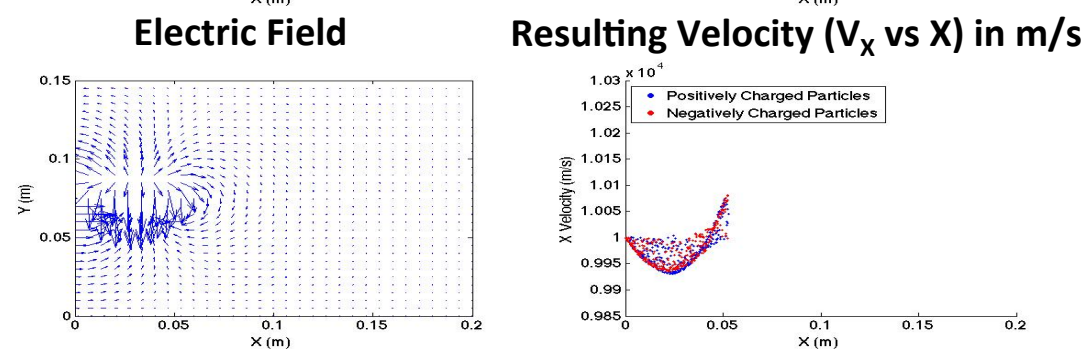

Figure 7. Beam Propagation of 50 nm Hollow Polystyrene Particles at Early Time Stage

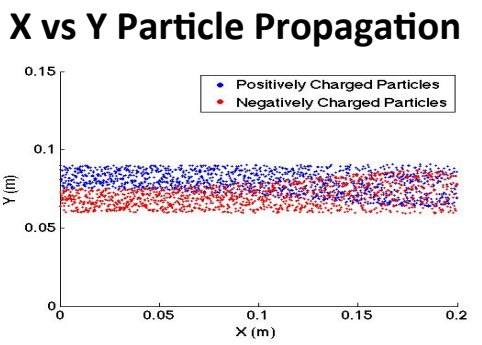

Electric Field

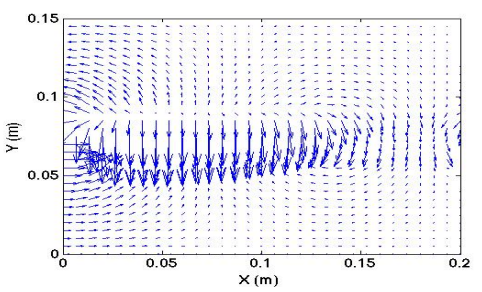

\section{Resulting Velocity $\left(V_{Y}\right.$ vs $\left.X\right)$ in $\mathrm{m} / \mathrm{s}$}

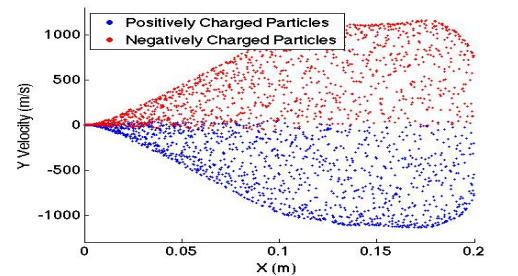

Resulting Velocity $\left(V_{x}\right.$ vs $\left.X\right)$ in $\mathrm{m} / \mathrm{s}$

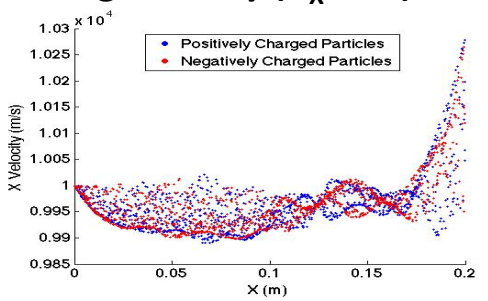

Figure 8. Beam Propagation of $50 \mathrm{~nm}$ Hollow Polystyrene Particles at Final Time Stage 
In this case we see similar results as the previous case which is the convergence of the two oppositely charged beams towards one another. We also see the image charge induced axial electric field that causes the initial emitted beam to be slowed down significantly by a maximum of $1.1 \%$. Again we see that as the beam continues to propagate across the simulation space the electric fields get stronger. Since these particles have a higher charge to mass ratio they move more easily, and thus you see the two beams more readily converge upon one another. You also see the larger slow down in velocity along with the larger increase in velocity on the leading edge. All of these results can easily be understood and compared to the previous simulation using a series of force equations as is seen in Eq. 2.

$$
q E=F=m a
$$

Changing this equation around gives Eq. 3.

$$
a=\frac{q}{m} E
$$

From this equation we can clearly see that with a higher specific charge, as we have in this case, the acceleration of the particles will increase. Since we are emitting the same amount of current, we will have similar initial electric fields, thus, the higher specific charge will result in both the axial velocity being slowed down more initially as well as reaching a higher leading edge velocity as well as the oppositely charged beams converging upon one another quicker.

\section{IV.C. Varying Beam Separation while Emitting 200 nm Solid Polystyrene Particles}

In these next two sets of simulations we vary beam separation and beam width to see how we can minimize the image charge induced axial electric field. In these simulations the simulation space and the emitter parameters are kept consistent as the two original time progression simulations. The simulation space is in Cartesian coordinates with $0.2 \mathrm{~m}$ in the $\mathrm{x}$ direction and $0.15 \mathrm{~m}$ in the $\mathrm{y}$ direction. Particles are emitted in the $\mathrm{x}$ direction. Again the current density for each emitted beam is kept at $0.967 \mathrm{~A} / \mathrm{m}^{2}$, with the emission being of $200 \mathrm{~nm}$ polystyrene particles as was used in the first simulation. In this simulation we vary the beam separation between two oppositely charged beams, while keeping the emission width constant at 1.5 $\mathrm{cm}$. We vary the beam separation from completely overlapping beams up to $8.5 \mathrm{~cm}$ between beam centers. Fig. 9, Fig. 10, and Fig. 11 show the beam propagation and diagnostics when the beam is almost to the end of the simulation space.

\section{$X$ vs $Y$ Particle Propagation Resulting Velocity $\left(V_{Y}\right.$ vs $\left.X\right)$ in $m / s$}

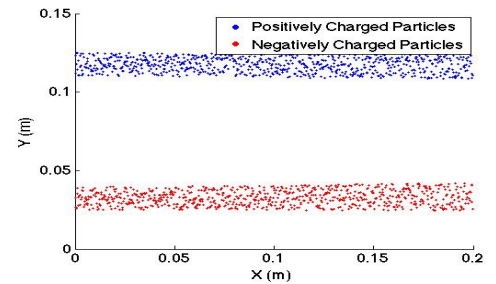

Electric Field
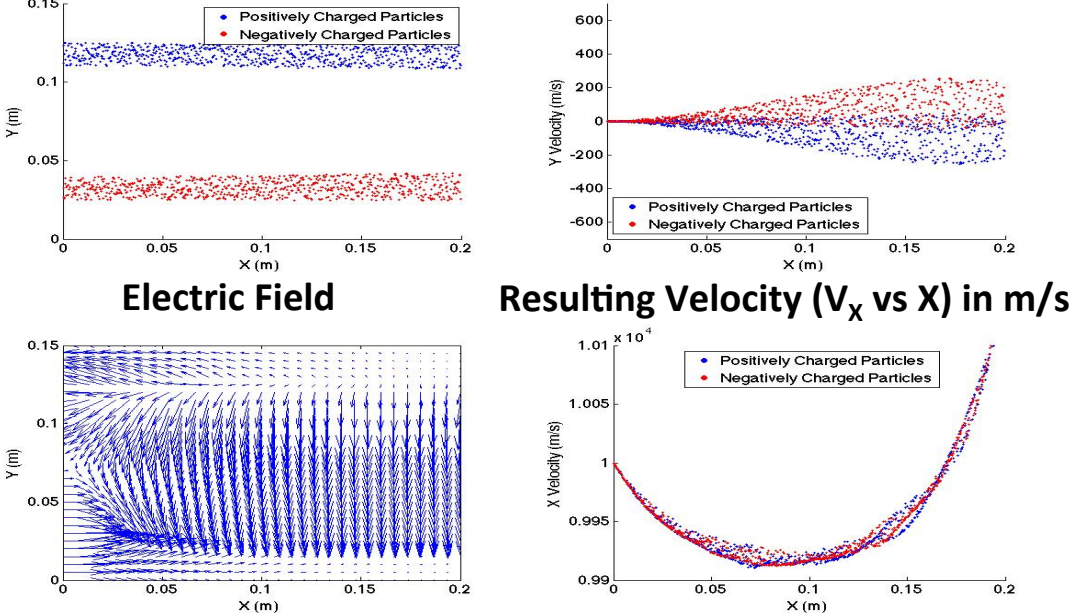

Figure 9. Beam Propagation and Diagnostics with a Beam Center Separation of $8.5 \mathrm{~cm}$ 
$X$ vs Y Particle Propagation Resulting Velocity $\left(V_{Y}\right.$ vs $\left.X\right)$ in $\mathrm{m} / \mathrm{s}$
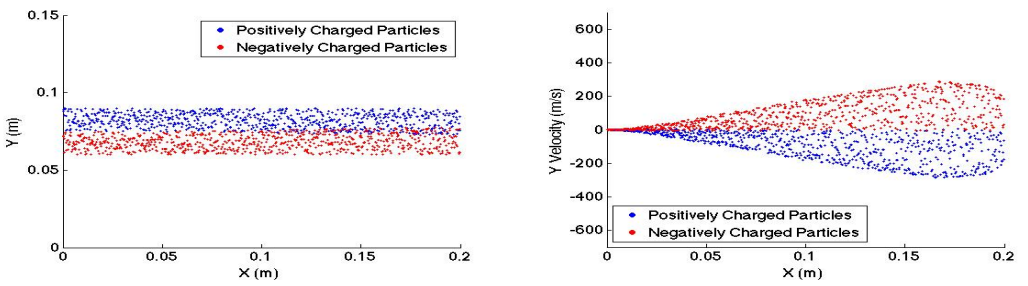

Electric Field

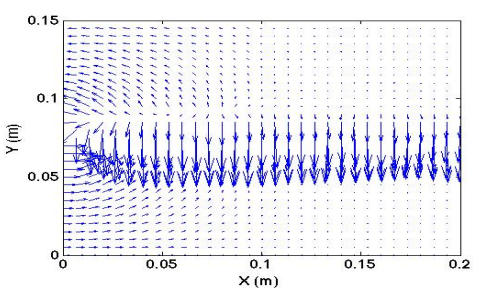

Resulting Velocity $\left(\mathrm{V}_{\mathrm{x}}\right.$ vs $\left.\mathrm{X}\right)$ in $\mathrm{m} / \mathrm{s}$

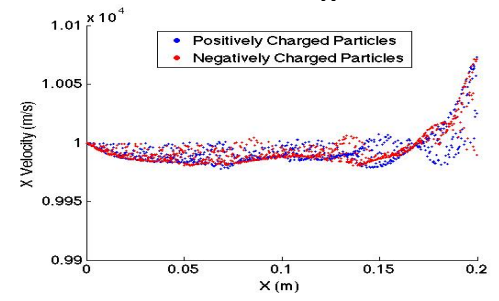

Figure 10. Beam Propagation and Diagnostics with a Beam Center Separation of $1.5 \mathrm{~cm}$

$X$ vs Y Particle Propagation Resulting Velocity $\left(V_{Y}\right.$ vs $\left.X\right)$ in $\mathrm{m} / \mathrm{s}$

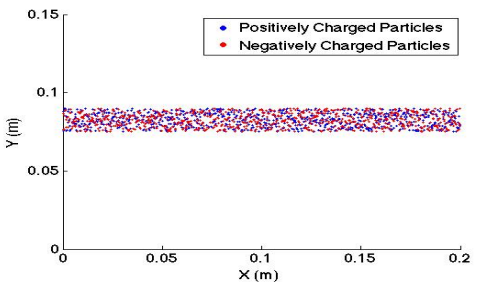

Electric Field

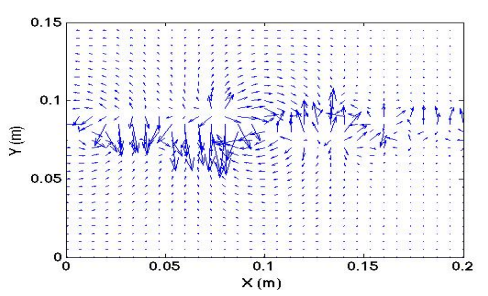

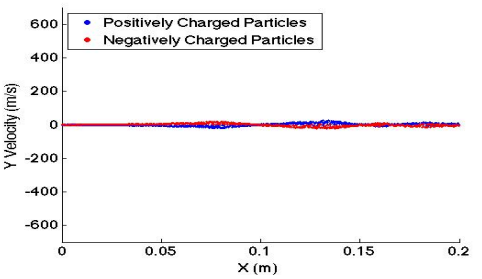

Resulting Velocity $\left(\mathrm{V}_{\mathrm{X}}\right.$ vs $\left.\mathrm{X}\right)$ in $\mathrm{m} / \mathrm{s}$

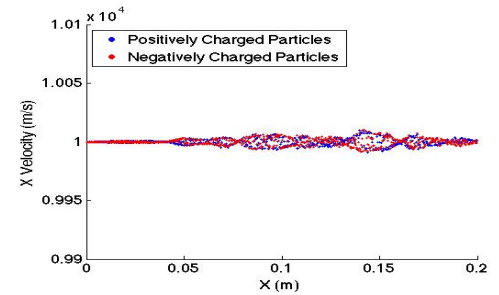

Figure 11. Beam Propagation and Diagnostics with a Beam Center Separation of $0 \mathrm{~cm}$ 
Fig. 9 shows a beam center separation of $8.5 \mathrm{~cm}$, Fig. 10 shows a beam center separation of $1.5 \mathrm{~cm}$ (which means that the beams are right next to one another), and Fig. 11 shows a beam center separation of $0 \mathrm{~cm}$ (which means that the beams are completely overlapping). In this case you see that as the beams are moved closer together, while keeping the beam widths constant, the image charge induced axial electric field decreases. This also means that the minimum velocity gets closer to the initial emission velocity as the beams are moved closer together, implying that there is less of an effect of the particles pulling back on the spacecraft negating the generated thrust. In this case we see a maximum velocity drop of $0.9 \%$ when the beams are $8.5 \mathrm{~cm}$ apart, and a velocity drop of just $0.2 \%$ when the beams are right next to one another. We also see that when the beams are completely overlapping, we have virtually no velocity drop because for all intents and purposes, the beam is completely neutral and there is no image charge induced on the spacecraft wall. This is what we consider a neutral emission beam, and a neutral spacecraft. However, this is difficult to generate in the NanoFET system because we cannot place emission beams exactly next to one another and alternate positive and negative emission holes due to electrical breakdown concerns.

\section{IV.D. Varying Beam Width while Emitting 200 nm Solid Polystyrene Particles}

Our next simulation is done by keeping beam separation constant (the two beams right next to one another), while varying the beam width from $0.5 \mathrm{~cm}$ up to $3.5 \mathrm{~cm}$. In this case we keep the simulation space and particles the same as the previous simulations. However, we must vary the current level in order to keep the current density for both beams constant at $0.966 \mathrm{~A} / \mathrm{m}^{2}$. In addition we need to change the number of particles that are represented by a simulated superparticle in order to keep the number of superparticles shown in the simulation constant. Fig. 12, Fig. 13, and Fig. 14 show the beam propagation and diagnostics as we decrease the beam width from $3.5 \mathrm{~cm}$ to $1.5 \mathrm{~cm}$ to $0.5 \mathrm{~cm}$. Again these simulations show that as beams get thinner, the image charge induced electric field continues to decrease, decreasing the drop in axial electric field from $0.9 \%$ for $3.5 \mathrm{~cm}$ wide beams to no velocity drop when the beams are $0.5 \mathrm{~cm}$ wide. This goes to show that as beam widths are reduced, the image charge induced axial electric field in the negative $\mathrm{x}$ direction gets weaker.

\section{$X$ vs Y Particle Propagation Resulting Velocity $\left(V_{Y}\right.$ vs $\left.X\right)$ in $\mathrm{m} / \mathrm{s}$}

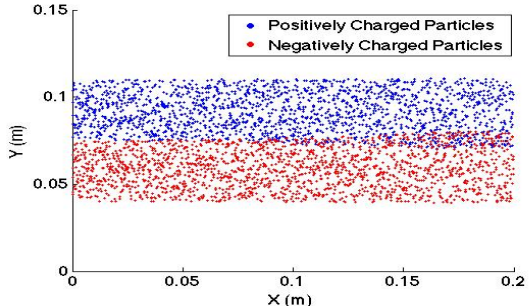

Electric Field

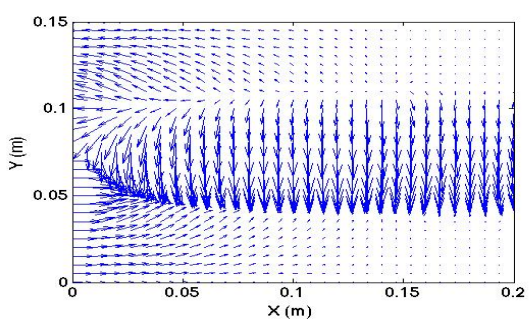

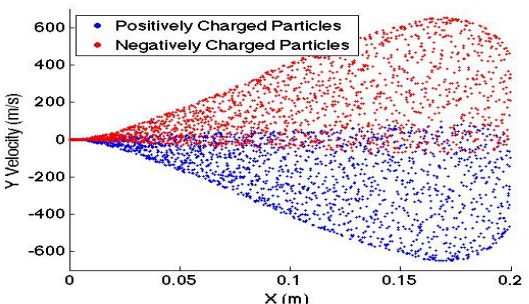

Resulting Velocity $\left(V_{X}\right.$ vs $\left.X\right)$ in $\mathrm{m} / \mathrm{s}$

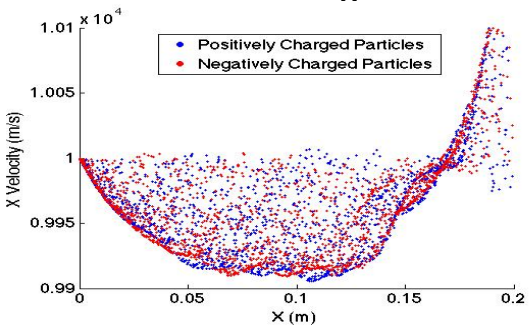

Figure 12. Beam Propagation and Diagnostics with a Beam Width of $3.5 \mathrm{~cm}$ 


\section{$X$ vs $Y$ Particle Propagation Resulting Velocity $\left(V_{Y}\right.$ vs $\left.X\right)$ in $\mathrm{m} / \mathrm{s}$}

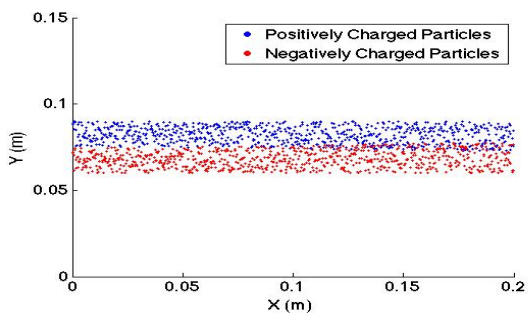

Electric Field

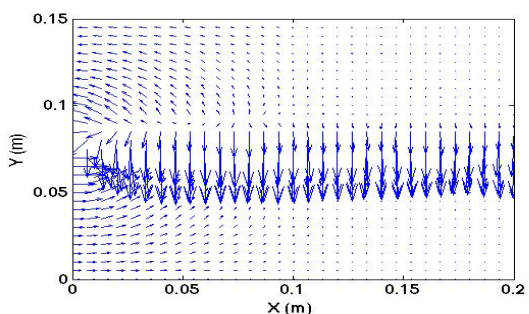

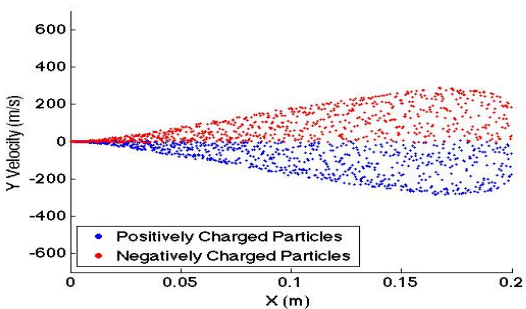

Resulting Velocity $\left(\mathrm{V}_{\mathrm{x}}\right.$ vs $\left.\mathrm{X}\right)$ in $\mathrm{m} / \mathrm{s}$

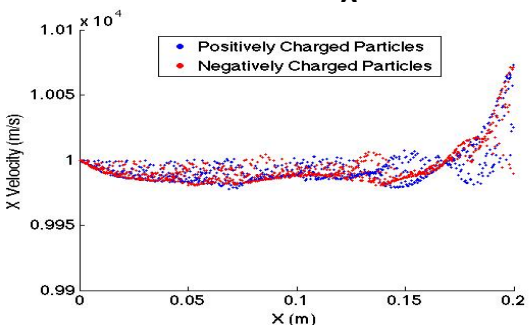

Figure 13. Beam Propagation and Diagnostics with a Beam Width of $1.5 \mathrm{~cm}$

\section{$X$ vs Y Particle Propagation Resulting Velocity $\left(V_{Y}\right.$ vs $\left.X\right)$ in $\mathrm{m} / \mathrm{s}$}

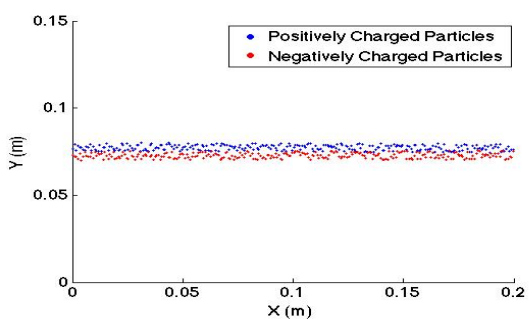

Electric Field

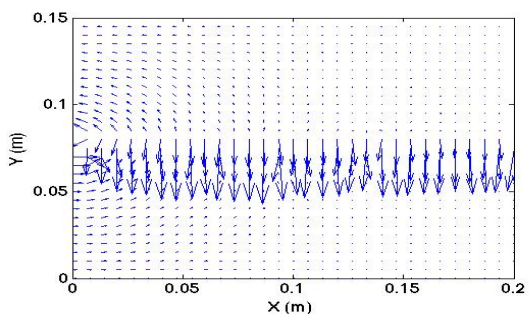

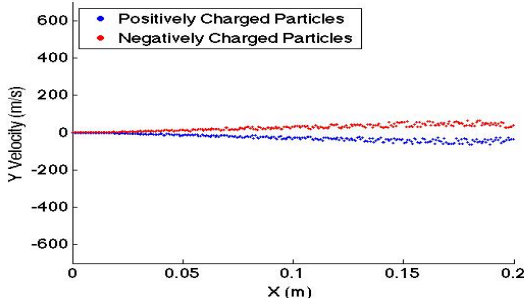

Resulting Velocity $\left(\mathrm{V}_{\mathrm{x}}\right.$ vs $\left.\mathrm{X}\right)$ in $\mathrm{m} / \mathrm{s}$

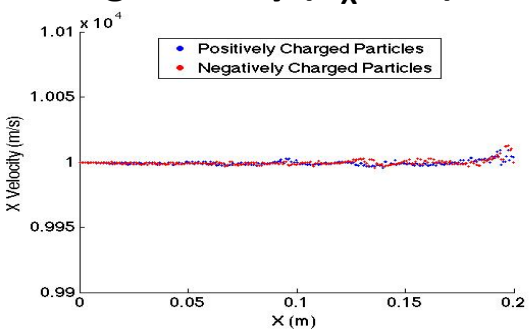

Figure 14. Beam Propagation and Diagnostics with a Beam Width of $0.5 \mathrm{~cm}$ 
These two simulations suggest that there are ways of mitigating the image charge induced axial electric field by moving the beams closer together as well as decreasing the widths of the beams. Combining these two effects along with increasing the number of alternating beams in the spatial-varying, common time emission scheme is similar to creating overlap of the two beams, which we also have shown to have a neutralizing effect. However, for use in the NanoFET system there are limits to which this can be done including how close oppositely charged gates can be placed, and how narrow these gates can be without creating undesirable arcing effects. This is an issue that is still of concern and will continue to be explored.

\section{IV.E. Varying Current Density while Emitting 200 nm Solid Polystyrene Particles}

Our last simulation shows the effects when current density is increased towards the space charge limit. As we have mentioned previously, the space charge limit in our simulation with a $1000 \mathrm{~s}$ specific impulse emitter, is approximately $680 \mathrm{~A} / \mathrm{m}^{2}$. Our previous simulations had been emitting particles with a current density of approximately $1 \mathrm{~A} / \mathrm{m}^{2}$. The following simulations show the results when the current density is increased to approximately $10 \mathrm{~A} / \mathrm{m}^{2}$, which is still well below the space charge limit. These simulations will have the same simulation space parameters as previous simulations with an emitter width of $1.5 \mathrm{~cm}$ and the beams placed adjacent to one another. As like in the varying beam width simulation set, as we vary current density, the number of particles being emitted will change as well; however, we again change the number of particles to superparticle ratio to keep the number of simulated particles constant. Fig. 15 and Fig. 16 show the diagnostics with a current density at 1 and $10 \mathrm{~A} / \mathrm{m}^{2}$ respectively.

\section{$X$ vs $Y$ Particle Propagation Resulting Velocity $\left(V_{Y}\right.$ vs $\left.X\right)$ in $\mathrm{m} / \mathrm{s}$}

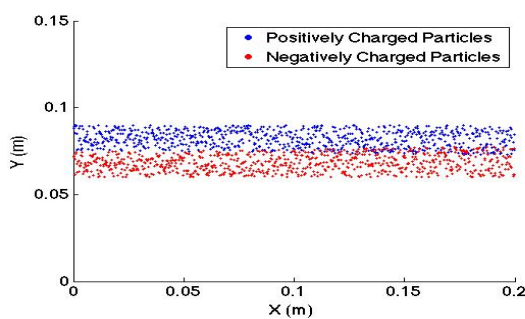

Electric Field

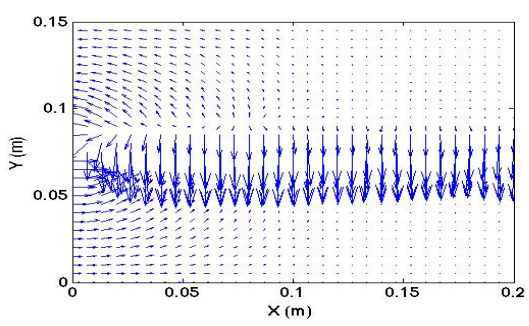

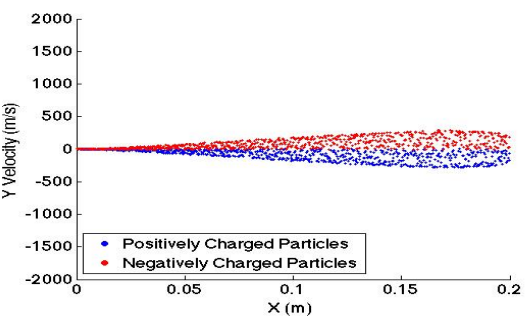

Resulting Velocity $\left(\mathrm{V}_{\mathrm{x}}\right.$ vs $\left.\mathrm{X}\right)$ in $\mathrm{m} / \mathrm{s}$

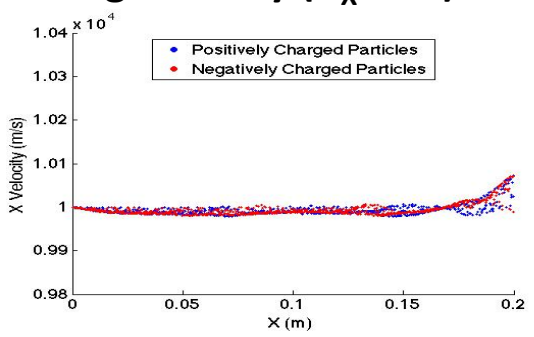

Figure 15. Beam Propagation and Diagnostics with a Current Density of $1 \mathrm{~A} / \mathrm{m}^{2}$ 


\section{$X$ vs Y Particle Propagation Resulting Velocity $\left(V_{Y}\right.$ vs $\left.X\right)$ in $\mathrm{m} / \mathrm{s}$}

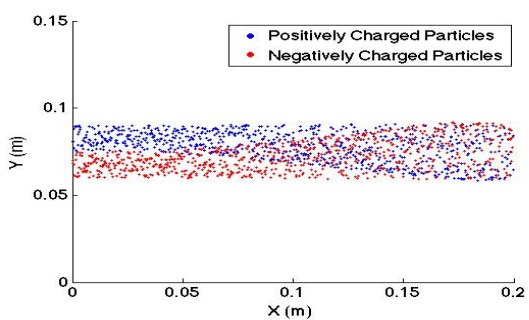

Electric Field

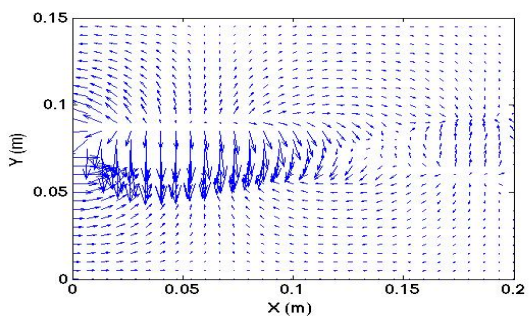

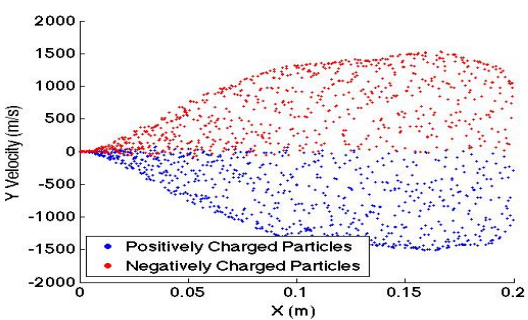

Resulting Velocity $\left(\mathrm{V}_{\mathrm{X}} \mathrm{vs} \mathrm{X}\right)$ in $\mathrm{m} / \mathrm{s}$

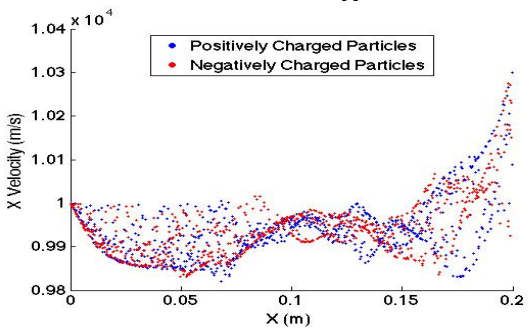

Figure 16. Beam Propagation and Diagnostics with a Current Density of $10 \mathrm{~A} / \mathrm{m}^{2}$

These two plots show clearly that as we increase current density by a factor of 10 , while we are well below space charge limit, the maximum drop in velocity increases a factor of ten from $0.22 \%$ to nearly $2 \%$. This shows that even though the maximum drop in velocity seemed insignificant in previous simulations, as we increase current density these effects will be very important and detrimental.

\section{Time-Varying, Common Spatial Emission Scheme}

Next, we examine the time-varying, common spatial emission scheme that was introduced earlier. It bears repeating that this is the scheme that has a single emission area that emits particles of a single polarity at any one time. However, the single emission area will change the polarity of particles that it emits over time in an oscillating manner. Over time, the beam and the spacecraft is net neutral; however, over short time scales both the beam and the spacecraft will charge up.

While we continue to develop a precise model for how the spacecraft will charge up as charged particles are emitted, we begin by using a simplified spacecraft charging model. This simplified spacecraft charging model will ignore the ionospheric plasma effects. This model assumes that the $3 \mathrm{U}$ cubesat that NanoFET will be placed on is a sphere rather than a rectangular prism. This sphere has a diameter equal to the length of the cubesat, thus giving it a diameter of $30 \mathrm{~cm}$ as shown in Fig. 17.

For a sphere, there are two ways to approach a formulation for correlating voltage that the sphere has charged up to, to the charge being expelled from the sphere, the first being Gauss' Law and the second being using capacitance of a sphere, with both giving us the same result. With Gauss' Law being more basic, we will use that approach starting with Gauss' Law in integral form as shown in Eq. 4.

$$
\oint E \cdot d A=\frac{Q}{\varepsilon_{0}}
$$

Knowing that it is a sphere meaning a constant radius we can transform this equation to Eq. 5.

$$
\frac{V}{r} \cdot 4 \pi r^{2}=\frac{Q}{\varepsilon_{0}}
$$

Giving us an equation as shown in Eq. 6.

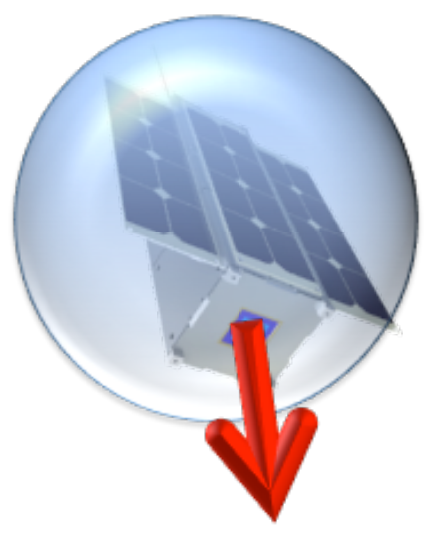

$$
V=\frac{Q}{4 \pi \varepsilon_{0} r}
$$

Figure 17. Cubesat Modeled by a $30 \mathrm{~cm}$ Diameter Sphere 
Lastly we know that current is simply flow of charge over time and thus can substitute in for charge and find a relationship for how quickly a spacecraft will charge up to certain voltages given a determined amount of current leaving the sphere as shown in Eq. 7.

$$
t=V \frac{4 \pi \varepsilon_{0} r}{I}
$$

From this equation we can determine how quickly our hypothetical $3 \mathrm{U}$ spherical cubesat will charge up to different percentages of the charging voltage of the NanoFET system. In this case we assume that the NanoFET system is charging and accelerating the particles out at $40 \mathrm{kV}$. Fig. 18 shows how quickly the simplified spacecraft will charge up to $1 \%, 5 \%, 10 \%$, and $20 \%$ of that charging and accelerating voltage depending on how much current we are emitting from the spacecraft. From this we can see that if we emit $14.5 \mathrm{~mA}$ of current as in previous simulations, the unneutralized $3 \mathrm{U}$ cubesat will charge up to $5 \%$ of the charging and accelerating voltage in under $10 \mu \mathrm{s}$. If we intend to keep the spacecraft charge below this $5 \%$ value, we will need to be oscillating between emitting positively and negatively charged particles at over 100 $\mathrm{kHz}$. Predictably, this is very difficult to do when we are charging the NanoFET system up to high voltage in one polarity in order to emit particles of a certain polarity, then quickly discharge and charge up to high voltage in the opposite polarity to emit the opposite charge. Thus, it seems like the time-varying, common spatial emission scheme will be very difficult to implement.

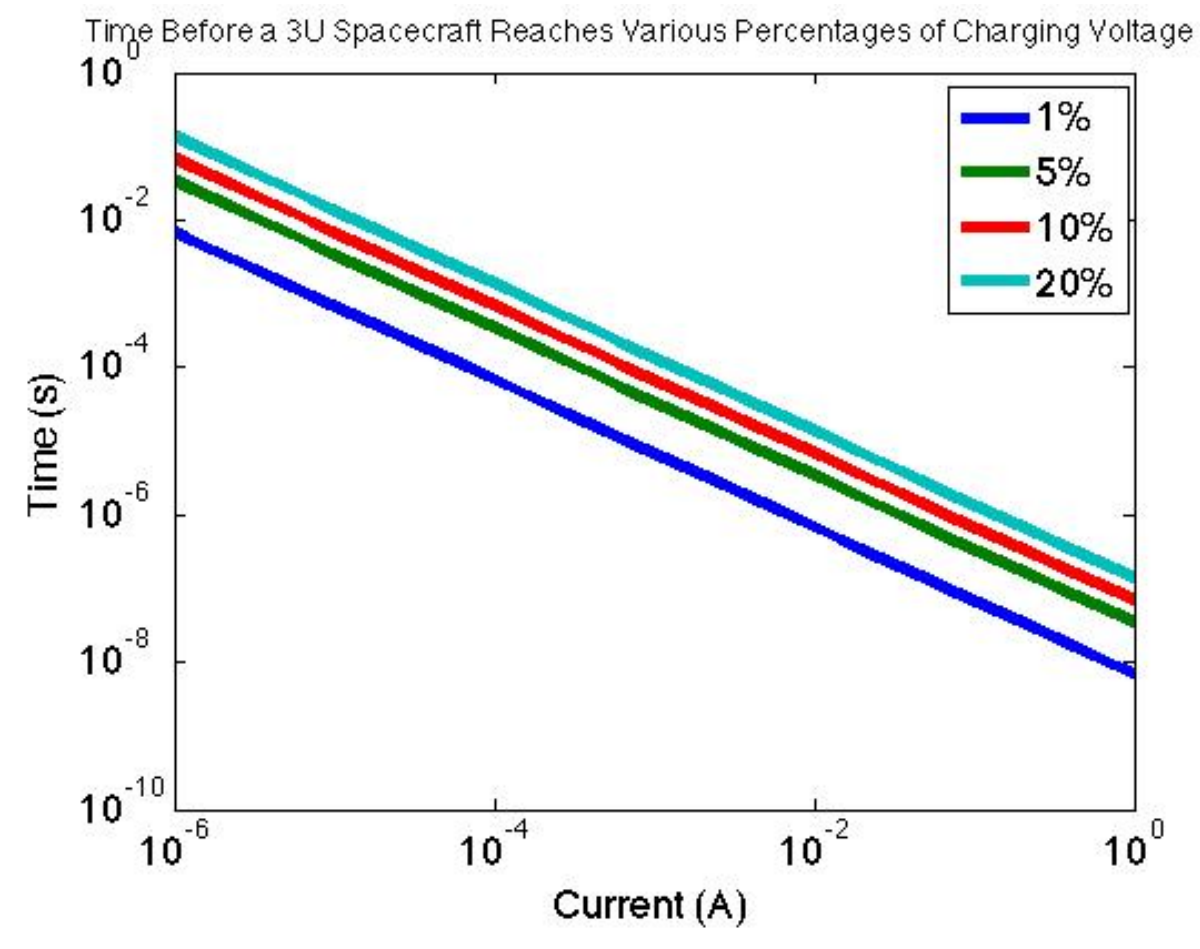

Figure 18. Time Before a 3U Spacecraft Reaches Various Percentages of Charging Voltage

\section{Conclusion}

From these simulations and models we can see that the two seemingly obvious neutralization methods, spatial-varying, common time emission and time-varying, common spatial emission, are not as easy to implement as originally assumed. In the case of the spatial-varying, common time emission scheme, even though the overall beam and the overall spacecraft are always both net neutral, local charge effects exist because we are using large, heavy particles to neutralize as opposed to light, fast electrons to neutralize as other systems use. There are ways to make this neutralization method possible. As we shrink the width of the beams, and as we place the oppositely charged beams closer to one another, the local charge effects are minimized. In order to utilize the full surface, rather than using just two beams like is shown in our 
simulations, we will use multiple beams alternating between positively and negatively charged beams. Thus, as we continue to shrink the beams and move them closer together, these beams will start to look like two oppositely charged beams stacked on top of one another which we have shown to have no local charge issues. However, there are constraints as to how close oppositely charged beams can be placed and how thin the beams can be as it increases the number of total beams needed. This is due to the high voltages that are necessary to charge and accelerate the particles, and concerns of arcing between the highly, oppositely charged gates. In the time-varying, common spatial emission scheme, we realized through our basic modeling that the spacecraft will charge up to a significant percentage of the charging and accelerating voltage in relatively short time scales when we are emitting significant amounts of current. Thus, this seems like a scheme that may not be feasible to implement without an additional neutralization technique.

\section{Acknowledgments}

D. C. Liaw thanks Jamin Rager for his help compiling this data along with Professor John Verboncoeur and his XOOPIC team for their help and advice with the modeling. Lastly, thanks go to Dr. Dan Brown and the Air Force Office of Scientific Research and Air Force Research Lab for their financial support of this work through the Michigan/Air Force Center of Excellence in Electric Propulsion.

\section{References}

\footnotetext{
${ }^{1}$ Domonkos, M.T., Gallimore, A.D., and Patterson, M.J., "An Evaluation of Hollow Cathode Scaling to Very Low Power and Flow Rate," International Electric Propulsion Conference, IEPC-97-189, University of Michigan, Ann Arbor, MI, 1997.

${ }^{2}$ Patterson, M.J., and Oleson, S.R., "Low-Power Ion Propulsion for Small Spacecraft," AIAA/ASME/SAE/ASEE Joint Propulsion Conference, AIAA-97-3060, NASA, Cleveland, OH, 1997.

${ }^{3}$ Gilchrist, B. E., Jensen, K.L., Gallimore, A.D., and Severns, J., "Space Based Applications For FEA Cathodes (FEACs)", Materials Issues in Vacuum Microelectronics: Symposium Proceedings, Materials Research Society, Warrendale, PA, V 621, p R481-R487, 2000.

${ }^{4}$ Temple, D., "Recent Progress in Field Emitter Array Development for High Performance Applications," Materials Science and Engineering, R24, pp. 185-239, 1999.

${ }^{5}$ Félici, N.J., "Forces et Charge de Petits Objets en Contact avec une Électrode Affectée d'un Champ Électrique," Revue Générale de l'Électricité, 75, pp. 1145-1160, 1966.

${ }^{6}$ Liu, T.M., "The Design Space of a Micro/Nano-Particle Electrostatic Propulsion System," Ph.D.Dissertation, Aerospace Engineering Dept., University of Michigan, Ann Arbor, MI, 2010.

${ }^{7}$ Verboncoeur, J.P., Langdon, A.B., and Gladd, N.T., "An Object-Oriented Electromagnetic PIC Code," Computer Physics Communications, 87, pp. 199-211, 1995.

${ }^{8}$ Birdsall, C.K., "Particle-in-Cell Charged-Particle Simulations, Plus Monte Carlo Collisions with Neutral Atoms, PICMCC," IEEE Transactions on Plasma Science, 19, 2, 1991.

${ }^{9}$ Luginsland, J.W., and Lau, Y.Y., "Virtual Cathode Formation due to Electromagnetic Transients," IEEE Transactions on Plasma Science, 26, pp. 901-904, 1998.

${ }^{10}$ Luginsland, J.W., Lau, Y.Y., Umstattd, R.J., and Watrous, J.J., "Beyond the Child-Langmuir Law: A review of recent results on multidimensional space-charge-limited flow," Physics of Plasma, Vol. 9, Issue. 5, pp. 2371-2376, 2002.
} 